\title{
Use of Systematic Review and Meta-Analysis in Environmental Health Epidemiology: a Systematic Review and Comparison with Guidelines
}

\author{
Mary C. Sheehan ${ }^{1} \cdot$ Juleen Lam $^{1}$ \\ Published online: 3 July 2015 \\ (C) The Author(s) 2015. This article is published with open access at Springerlink.com
}

\begin{abstract}
Systematic review (SR) and meta-analysis (MA) have potential to contribute substantially to environmental health $(\mathrm{EH})$ risk assessment and policy-making, provided study questions are clear and methods sound. We undertook a systematic review of the published epidemiological literature for studies using both SR and MA examining associations between chronic low-dose chemical exposures and adverse health outcomes in general populations and compared actual methods and reporting with a checklist based on available published guidelines. We identified 48 EH SRMAs meeting these criteria. Associations were mainly positive and statistically significant, often involving large populations. A majority of studies followed most general SRMA guidance, although we identified weaknesses in problem formulation, study search, selection and data extraction, and integrating policy implications. Fewer studies followed EH-specific SRMA recommendations, particularly regarding exposure heterogeneity and other risks of bias. Development and adoption of EHspecific SRMA guidelines would contribute to strengthening these tools for public health decision-making.
\end{abstract}

This article is part of the Topical Collection on Global Environmental Health and Sustainability

Electronic supplementary material The online version of this article (doi:10.1007/s40572-015-0062-z) contains supplementary material, which is available to authorized users.

Mary C. Sheehan

msheeh10@jhu.edu

1 Department of Health Policy and Management, Johns Hopkins Bloomberg School of Public Health, 615 North Wolfe Street, Baltimore, MD 21205, USA
Keywords Systematic review $\cdot$ Meta-analysis · Environmental health $\cdot$ Reporting guidelines $\cdot$ Air pollution . Environmental epidemiology

\section{Introduction}

The World Health Organization (WHO) estimates that environmental factors are responsible for at least one quarter of the global burden of disease [1]. A growing body of evidence suggests a considerable share stems from exposure to environmental chemicals [2]; in many cases, such exposures are widespread across large populations or involve vulnerable groups [2,3]. Preventing environmentally related disease requires the translation of up-to-date scientific research into accessible evidence that informs environmental health (EH) risk assessment, policy formulation, health care practice, and individual health behaviors $[4,5]$. However, the scientific literature is diverse, of varying quality, and often not easily accessible to decision-makers [6].

The tools of evidence-based science - systematic review (a literature review that poses a well-defined and specific research question, uses systematic and explicit methods to identify, select and appraise research, and analyze data from selected studies) and metaanalysis (a quantitative statistical analysis that integrates results of chosen studies) [7] — are well-developed for randomized controlled trials and making recommendations in clinical medicine. These tools also have the potential to play a role in EH risk assessment and policy-making. When used together, systematic review and meta-analysis (SRMA) techniques provide rigorous rules designed to gather all relevant research on a welldefined study question, statistically pool outcomes 
across studies with comparable methods, and provide a transparent, thorough, and replicable examination of available evidence. This can help to overcome the drawbacks of small sample size, demonstrate where effects are consistent across studies and generalizable across populations and where they are not, minimize bias and reduce chance effects, and identify research gaps [8]. EH science evidence with these features can help facilitate efficient policy-making.

Human epidemiological studies are the preferred body of evidence for $\mathrm{EH}$, as they represent real-world background and co-exposures and do not require species extrapolation. However, in practice, the observational designs of human epidemiological studies in EH pose challenges to conducting MA that have contributed to slower adoption of SRMA. Among these are inability to fully control for confounders at all times, inconsistencies across studies in exposure measurement methods and metrics, and differences in outcomes, populations, and study designs [9]. Development of special metaanalytical methods to control for confounding [10] and risk-of-bias assessment checklists [11] have contributed to improving SRMA methods for observational studies. Codification of reporting guidance, such as the MetaAnalysis of Observational Studies in Epidemiology (MOOSE) consensus statement [12], has also advanced the field; use of such guidelines is associated with higher quality reporting [13]. More recently, the US National Institutes of Environmental Health Sciences (NIEHS) National Toxicology Program (NTP) has adopted SRMA in its chemical assessments [14•]; the US EPA is examining using these tools for risk assessment under its Integrated Risk Information System (IRIS) program [15•]; and the Navigation Guide group (an interdisciplinary collaboration between academicians, practitioners, and clinicians) has developed a framework to assess the strength of $\mathrm{EH}$ science evidence $[16,17 \bullet]$. These newer initiatives are contributing to the refinement and codification of methodological approaches for SRMA tailored to the specificities of EH.

The potential of SRMA to contribute to EH policy, the development of more robust, EH-specific methods, and growing interest in their use suggest the need to take stock of the nature and quality of the current body of published EH SRMAs. We undertook a systematic review of the EH literature to identify SRMA studies examining associations between chronic low-dose chemical exposures and adverse health outcomes in general human populations in order to compare methods used with available published consensus reporting guidelines. The broad goal of this review is to contribute to enhancing the utility and expanding use of these powerful evidence-synthesis tools for EH policy.

\section{Methods}

We conducted a search of the published scientific literature using Medline, Embase, Web of Science, Scopus, and Google Scholar (with the search terms "environment" OR "pollutant" OR "contaminant" AND "disease" OR "chronic AND disease" OR "health AND effects" AND "systematic" AND "review" AND "meta-analysis" OR "meta AND analysis" OR "quantitative AND analysis," screened for human studies only). We performed topic searches in these electronic databases examining keywords, titles, and abstracts for designated terms and also hand-searched reference lists of selected studies. We did not restrict by language or starting date. Our search end date was June 30, 2013. We screened all titles and abstracts identified and reviewed full texts of articles that met prespecified inclusion criteria. We included only studies employing both SR and MA. We did not include SRs done without MA (e.g., SRs done as scoping reviews, SRs without quantitative outcomes, or SRs where reported outcomes were inadequate for a statistical analysis) or MAs for which SR had not been conducted (e.g., MA used to combine results across multicenter epidemiological studies or dose-response assessment using MA based on a few specifically chosen studies rather than a SR). We included SRMAs examining general, non-occupationally exposed populations exposed to chemicals at chronic, low dose (excluding biological, mental, or physical exposures, accidental high-dose acute exposures, intentional exposures such as alcohol or tobacco, and secondhand smoke exposure) that examined associations with one or more adverse health effects.

Both authors independently extracted data from included studies (differences were resolved by consensus), using purpose-designed data extraction forms previously tested on a pilot group of reviews. Extracted data included study goal, population characteristics, environmental exposures and their measurement protocols, health outcomes and their ascertainment procedures, summary effect measures (with confidence bounds), and responses to a checklist of 61 parameters of study methodological and reporting quality. We developed the checklist based on guidelines for SRMAs available to researchers during our review period, including the Preferred Reporting Items for Systematic Reviews and Meta-Analyses (PRISMA) statement [7], the MOOSE statement [11], and guidelines for use of SRMA in environmental epidemiology resulting from a 1994 workshop (referred to as "Blair et al.") [18]. Checklist items for the general features of SRMA were based primarily on the PRISMA and MOOSE statements, while those related to quality and risk-of-bias evaluation, heterogeneity testing, exposure measurement, and outcome ascertainment were also based on Blair et al. The checklist questions were designed to have up to five possible responses: yes $(\mathrm{Y})$, consistent with guideline; partial $(\mathrm{P})$, in some part consistent with guideline; no $(\mathrm{N})$, inconsistent with guideline; 
cannot determine based on data (ND); and not applicable to study (NA). For each parameter, definitions corresponding to guideline recommendations were developed beforehand to facilitate consistency in reviewer data extraction (available in Supplement 1). Responses were coded and combined in Microsoft Excel and transferred to STATA version 10.0 (StataCorp, College Station, TX).

\section{Results}

Our search identified 1136 articles, of which the full texts for 146 were reviewed and 48 were selected (Fig. 1 and Table 1, details in Supplement 1). All except two SRMAs were published after 2000, the year the MOOSE guidelines were issued; two thirds were published after 2009, the year the PRISMA guidelines were published (Fig. 2). Selected reviews covered a total of 16 chemicals or classes of chemicals, in five categories: (i) 11 reviews of indoor air pollution (IAP), consisting of various types of solid fuel smoke [19-29]; (ii) 12 reviews of various outdoor air pollution (OAP) constituents [30-41]; (iii) 10 reviews of metals [42-51]; (iv) seven reviews of persistent organic pollutants (POPs) [52-58]; and (v) eight reviews of other chemicals [59-66].

Associations with 28 health outcomes were explored, in five categories: (i) cancers, including bladder, breast, colorectal, lung, prostate, and childhood leukemia ( $27 \%$ of reviews); (ii) non-cancer respiratory diseases, including childhood asthma, childhood pneumonia, chronic obstructive pulmonary disease (COPD), tuberculosis, and acute respiratory infection (21\%); (iii) reproductive and development disorders, including birth defects, poor birth outcomes, and developmental neurotoxicity (19 \%); (iv) cardiovascular disease (CVD), including hypertension, myocardial infarction, stroke, and heart rate variability (19\%); and (v) other health outcomes, including Parkinson's disease, multiple sclerosis, and general mortality (14\%).

Adults were assessed in $63 \%$ of SRMAs, while infants or children alone or in combination with women were the focus of $37 \%$ of reviews. Most reviews did not limit geographic coverage; however, the majority of underlying study populations were from North America, Europe, and/or Asia. Thirtyeight percent of reviews examined primarily (but not exclusively) case-control studies, $27 \%$ primarily cohort studies, $16 \%$ primarily cross-sectional studies, $6 \%$ time series and case-crossover studies, and $13 \%$ examined a mix in which no study design was preponderant. Sixty percent of selected SRMAs reported pooled odds ratios (ORs); one quarter reported pooled relative risks (RRs), and the remainder reported other measures. Information on funding sources was provided in $67 \%$ of SRMAs. Where this information was provided, reviews were sponsored either by academic organizations (63\%) or government bodies (37\%). Conflict of interest statements were provided by about half of studies; most of these declared no conflict.

\section{SRMA Findings by Chemical Category}

Indoor Air Pollution The IAP SRMAs examined exposures due to smoke from solid fuels (coal, wood, animal dung, crop wastes) used in cooking and heating, mainly in Africa, Latin America, and Asia, with one study examining formaldehyde exposure. Nearly three quarters examined respiratory outcomes, and one quarter investigated cancers (mainly lung cancer) or other outcomes (Table 1). Among statistically significant findings of the largest magnitude, three SRMAs examining coal smoke exposure and lung cancer in adults reported ORs of 2.15 (95 \% CI 1.61, 2.89, $n=25)$ [20], $1.82(95 \%$ CI $1.60,2.06, n=23$ ) [23], and 2.66 (95 \% CI 1.39, 5.07, $n=8)$ [25]. Three reviews of solid fuel smoke exposure and COPD in adults found pooled ORs of 2.44 (95\% CI 1.79, 3.33, $n=$ 15) [21], $2.80(95 \%$ CI 1.85, 4.00, $n=12)$ [22], and 2.40 (95\% CI 1.47, 3.93, $n=6$ ) [25]. Two reviews of children $<5$ years old examining solid fuel smoke exposure and respiratory infection found combined ORs of 2.51 (95\% CI 1.53, $4.10, n=9$ ) [24] and 3.53 (95\% CI 1.94, 6.43, $n=10$ ) [25], while one review of solid fuel smoke and childhood pneumonia found an OR of 1.79 (95\% CI 1.26, 2.21, $n=24)$ [19].

Outdoor Air Pollution The OAP SRMAs examined exposures including carbon monoxide $(\mathrm{CO})$, ozone $\left(\mathrm{O}_{3}\right)$, sulfur dioxide $\left(\mathrm{SO}_{2}\right)$, nitrogen dioxide $\left(\mathrm{NO}_{2}\right)$, particulate matter $<2.5 \mu \mathrm{m}\left(\mathrm{PM}_{2.5}\right)$ and $<10 \mu \mathrm{m}\left(\mathrm{PM}_{10}\right)$ in various combinations in large urban areas. Over $40 \%$ evaluated CVD outcomes, and the remainder respiratory, and reproductive and development outcomes (Table 1). Among statistically significant associations of largest magnitude, an incremental $10 \mu \mathrm{g} / \mathrm{m}^{3}$ of $\mathrm{PM}_{2.5}$ was associated with various cardiovascular outcomes, including cardiovascular mortality (RR 1.15; $95 \%$ CI 1.04, $1.27 ; n=10$ ) [31]; heart failure (RR $1.02 ; 95 \%$ CI 1.01, 1.02 , $n=10$ ) [36]; myocardial infarction (RR 1.03; $95 \%$ CI 1.02, $1.04 ; n=13$ ) [34]; and lower heart rate variability (decrease of $2.44 \%, 95 \%$ CI $3.76 \%, 1.12 \%, n=13)$ [35].

Metals The metal SRMAs covered exposure to arsenic, lead, manganese, and mercury through various exposure pathways, mainly in the USA and Asia. Forty percent of reviews examined cardiovascular outcomes and the remainder a range of others (Table 1). Among statistically significant associations of the largest magnitude were arsenic exposure in drinking water (comparing high to low dose) and various cardiovascular-related outcomes, including CVD (RR 1.32; $95 \%$ CI 1.05, 1.67, $n=18$ ) [47]; diabetes type 2 (OR 2.52; $95 \%$ CI 1.69, 3.75, $n=6$ ) [48] (this meta-analysis was restricted to populations with high arsenic exposure from Taiwan and 


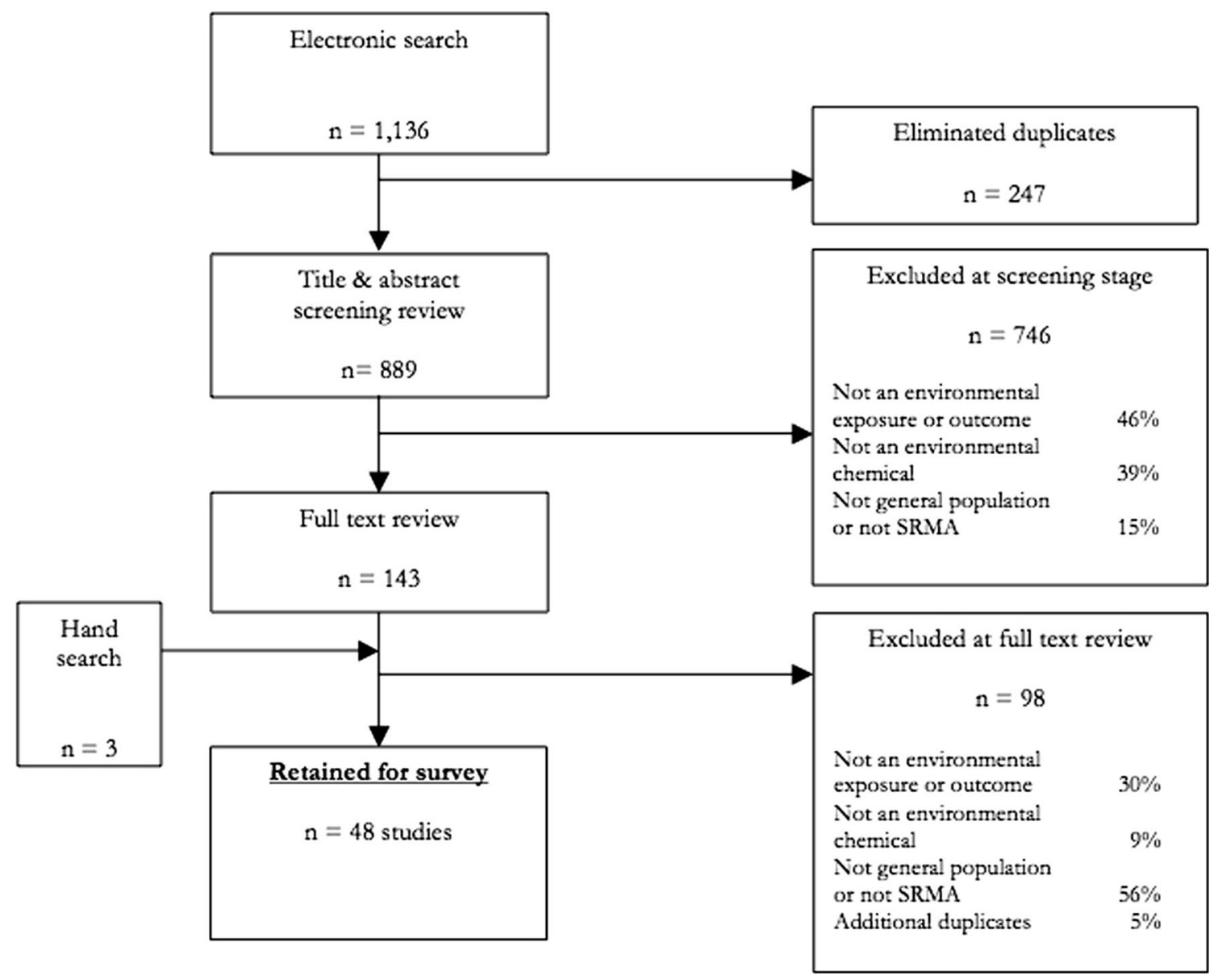

Fig. 1 Systematic review of environmental health (EH) epidemiology systematic review and meta-analyses (SRMA): search and selection flow diagram

Bangladesh), and hypertension (OR 1.27; $95 \%$ CI 1.09, 1.47, $n=8)$ [42].

Persistent Organic Pollutants The POP SRMAs covered residential pesticide exposure, the DDT metabolite DDE, and mixed POPs. Nearly $70 \%$ of reviews evaluated cancers and the remainder other outcomes (Table 1). Among statistically significant associations of largest magnitude, three reviews of residential pesticide exposure in utero and childhood leukemia found pooled ORs of 1.30 (95\% CI 0.86, 1.97, $n=4)$ [53] and 2.05 (95\% CI 1.80, 2.32, $n=11)$ [56] and RR of 2.19 ( $95 \%$ CI 1.92, 2.50, $n=13$ ) [57]. One review found an apparent lack of association between DDE and breast cancer in women (OR 0.97; $95 \%$ CI 0.87, 1.09, $n=21$ ) [54].

Other Chemicals The other chemical SRMAs examined exposure to disinfection by-products and fluoride in drinking water and exposure to hair dye. Nearly $70 \%$ of reviews examined cancers, and the remainder involved other outcomes (Table 1). Among observed statistically significant associations of largest magnitude were disinfection by-products and various cancers, including of the bladder (RR 1.21; $95 \% \mathrm{CI}$ $1.09,1.34, n=7$; and OR 1.4; $95 \%$ CI $1.2,1.7, n=8)[62,66]$; rectum (RR 1.4; $95 \%$ CI 1.2, 1.7, $n=6$; and RR $1.30 ; 95 \%$ CI $1.06,1.59, n=10)[62,64]$; and colon (RR 1.27; $95 \%$ CI 1.08, $1.50, n=10)$ [64]. In addition, one SRMA found a null association between disinfection by-products and both low birth weight (RR 1.0; $95 \%$ CI $0.97,1.03, n=4$ ) and small for gestational age (RR $1.0195 \%$ CI 1.00, 1.02, $n=6$ ) [60].

\section{Comparison of Methods to Guidelines}

Study Background and Goal As shown in Supplement 2, the vast majority of reviews (94\%) outlined the public health problem and identified a study goal or research question; however, just $54 \%$ defined the goal or question sufficiently clearly to identify all recommended elements (population, health outcome, chemical exposure, and type of study) of a goal statement. Only $40 \%$ of titles self-identified as both SR and MA (an additional $50 \%$ identified as one or the other). One quarter referred to the use of an SRMA guideline; all of these referred either to the PRISMA or MOOSE statements. Only $8 \%$ of reviews reported having an ex-ante study protocol or plan.

Search, Selection, and Extraction Virtually all reviews reported on the electronic databases used to identify studies. Of 
Table 1 Systematic review of environmental health (EH) epidemiology systematic review and meta-analyses (SRMA): characteristics of selected studies

\begin{tabular}{|c|c|c|c|c|c|}
\hline Study reference & Chemical & Category & Health outcome & Population & Pooled estimate $^{\mathrm{a}}$ \\
\hline \multicolumn{6}{|l|}{ Indoor air pollution (IAP) } \\
\hline Zhao et al. 2006 & Coal smoke & CANC & Lung & Adults & OR $2.66(1.39,5.07)$ \\
\hline Hosgood et al. 2011 & Coal smoke & CANC & Lung & Adults & OR $2.15(1.61,2.89)$ \\
\hline Kurmi et al. 2012 & Coal smoke & CANC & Lung & Adults & OR $1.82(1.60,2.06)$ \\
\hline Dherani et al. 2008 & Solid fuel smoke & RESP & Pneumonia & Children $<5$ & OR $1.78(1.45,2.18)$ \\
\hline Kurmi et al. 2010 & Solid fuel smoke & RESP & COPD & Adults & OR $2.80(1.85,4.00)$ \\
\hline McGwin et al. 2010 & Formaldehyde & RESP & Asthma & Children & OR $1.17(1.01,1.36)$ \\
\hline Hu et al. 2010 & Biomass smoke & RESP & COPD & Adults & OR $2.44(1.79,3.33)$ \\
\hline Po et al. 2011 & Biomass smoke & RESP & ARI & Children $<5$ & OR $3.53(1.94,6.43)$ \\
\hline Misra et al. 2012 & Solid fuel smoke & RESP & ARI & Children $<5$ & OR $2.51(1.53,4.10)$ \\
\hline Sumpter et al. 2013 & Solid fuel smoke & RESP & Tuberculosis & Adults & OR $1.30(1.04,1.62)$ \\
\hline Pope et al. 2010 & Solid fuel smoke & REPDEV & Low birth weight & Infants & OR $1.38(1.25,1.52)$ \\
\hline \multicolumn{6}{|l|}{ Outdoor air pollution (OAP) } \\
\hline Chen et al. 2008 & $\mathrm{PM}_{2.5}$ & CANC & Lung & Adults & RR $1.21(1.10,1.32)$ \\
\hline Mustafic et al. 2011 & $\mathrm{PM}_{2.5}$ & CVD & Myocardial infarction & Adults & RR $1.03(1.02,1.04)$ \\
\hline Pieters et al. 2012 & $\mathrm{PM}_{2.5}$ & CVD & Heart rate variability & Adults & $\%-2.44(-3.76,-1.12)$ \\
\hline Li et al. 2012 & $\mathrm{PM}_{2.5}$ & CVD & Stroke & Adults & RR $1.01(1.00,1.01)$ \\
\hline Shah et al. 2013 & $\mathrm{PM}_{2.5}$ & CVD & Heart failure & Adults & RR $1.016(1.008,1.023)$ \\
\hline Hoek et al. 2013 & $\mathrm{PM}_{2.5}$ & CVD & CVD mortality & Adults & RR $1.15(1.04,1.27)$ \\
\hline Shang et al. 2013 & $\mathrm{PM}_{2.5}$ & RESP & Respiratory mortality & Adults & RR $0.51(0.30,0.73)$ \\
\hline Ward et al. 2004 & $\mathrm{PM}_{2.5}$ & RESP & Peak expiratory flow & Children & $\%-0.144(-0.243,-0.044)$ \\
\hline Weinmayr et al. 2010 & $\mathrm{NO}_{2}$ & RESP & Asthma symptoms & Children & OR $1.031(1.001,1.062)$ \\
\hline Vrijheid et al. 2011 & $\mathrm{NO}_{2}$ & REPDEV & Congenital anomalies & Infants & OR $1.20(1.02,1.42)$ \\
\hline Stieb et al. 2012 & $\mathrm{PM}_{10}$ & REPDEV & Low birth weight & Infants & OR $1.10(1.05,1.15)$ \\
\hline Ito et al. 2005 & Ozone & OTHER & All-cause mortality & Adults & $\% 1.6(1.1,2.0)$ \\
\hline \multicolumn{6}{|l|}{ Metals } \\
\hline Chu et al. 2006 & Arsenic & CANC & Bladder & Adults & Slope factor $3 \times 10^{-5}$ \\
\hline Mink et al. 2008 & Arsenic & CANC & Bladder & Adults & RR $1.11(0.95,1.30)$ \\
\hline Navas-Acien et al. 2008 & Lead & CVD & Hypertension & Adults & OR $1.04(1.01,1.07)$ \\
\hline Gallagher et al. 2010 & Cadmium & CVD & Diastolic BP & Women & Beta $1.84(0.95,2.74)$ \\
\hline Abhyankar et al. 2011 & Arsenic & CVD & Hypertension & Adults & OR $1.27(1.09,1.47)$ \\
\hline Moon et al. 2012 & Arsenic & CVD & Multiple CVD outcomes & Adults & RR $1.32(1.05,1.67)$ \\
\hline Pocock et al. 1994 & Lead & REPDEV & IQ point loss & Children $<5$ & $-2.53(-3.33,-1.73)$ \\
\hline Rodriguez-Barranco et al. 2013 & Arsenic & REPDEV & IQ point loss & Children $<5$ & $-0.39(-0.84,0.06)$ \\
\hline Navas-Acien et al. 2006 & Arsenic & OTHER & Diabetes type 2 & Adults & OR $2.52(1.69-3.75)$ \\
\hline Aminzadeh et al. 2007 & Mercury & OTHER & Multiple sclerosis & Adults & OR $1.24(0.96,1.61)$ \\
\hline \multicolumn{6}{|l|}{ Persistent organic pollutants (POPs) } \\
\hline Lopez-Cervantes et al. 2004 & DDE & CANC & Breast & Women & OR $0.97(0.87,1.09)$ \\
\hline Turner et al. 2010 & Pesticides & CANC & Child leukemia & Children $<5$ & OR $1.54(1.13,2.11)$ \\
\hline Van Maele-Fabry et al. 2011 & Pesticides & CANC & Child leukemia & Children $<5$ & OR $1.74(1.37,2.21)$ \\
\hline Bailey et al. 2011 & Pesticides & CANC & Child leukemia & Children $<5$ & OR $1.37(1.00,1.88)$ \\
\hline Priyadarshi et al. 2001 & Pesticides & OTHER & Parkinson's disease & Adults & OR $1.85(1.31,2.60)$ \\
\hline Allen et al. 2013 & Pesticides & OTHER & Parkinson's disease & Adults & OR $1.36(1.05,1.75)$ \\
\hline Wu et al. 2013 & PCBs & OTHER & Diabetes type 2 & Adults & $1.70(1.28,2.27)$ \\
\hline \multicolumn{6}{|l|}{ Other } \\
\hline Morris et al. 1992 & DBP & CANC & Bladder & Adults & OR $1.21(1.09,1.34)$ \\
\hline Villanueva et al. 2003 & DBP & CANC & Bladder & Adults & OR $1.4(1.2,1.7)$ \\
\hline Takkouche et al. 2005 & Hair dye & CANC & Hemopoietic & Adults & RR $1.15(1.05,1.27)$ \\
\hline
\end{tabular}


Table 1 (continued)

\begin{tabular}{llllll}
\hline Study reference & Chemical & Category & Health outcome & Population & Pooled estimate $^{\text {a }}$ \\
\hline Rahman et al. 2010 & DBP & CANC & Rectal & Adults & RR 1.30 (1.06, 1.59) \\
Hwang et al. 2003 & DBP & REPDEV & Any birth defects & Infants & OR 1.25 (1.11, 1.40) \\
Nieuwenhuijsen et al. 2009 & DBP & REPDEV & Any birth defects & Infant & OR 1.17 (1.02, 1.34) \\
Grellier et al. 2010 & THM & REPDEV & SGA & Infants & OR 1.01 (1.00, 1.02) \\
Choi et al. 2013 & Fluoride & REPDEV & IQ point loss & Infants & Diff. $-0.45(-0.56,-0.35)$ \\
\hline
\end{tabular}

${ }^{\text {a }}$ Pooled effect sizes shown are the principal chemical exposure and health effect associations reported in reviews (where more than one association was examined, the association shown is that with the largest number of underlying studies or with the strongest identified association among those with similar numbers of studies). Fuller results are reported in Supplemental Material File 1. Estimates compare exposed with unexposed or higher with lower exposure groups, or provide risk per unit of pollutant

$A R I$ acute respiratory infection, $C A N C$ cancer, $C O P D$ chronic obstructive pulmonary disease, $C V D$ cardiovascular disease, $D D E$ dichlorodiphenyldichloroethylene, $D B P$ disinfection by-products, $I Q$ intelligence quotient, $\mathrm{NO}_{2}$ nitrogen dioxide, $P C B s$ polychorinated biphenyls, $P M_{2.5}$ particulate matter $<2.5 \mu \mathrm{m}, R E P D E V$ reproductive/developmental, $R E S P$ respiratory, $S G A$ small for gestational age, $T H M$ trihalomethane

these, $62 \%$ conducted a wide search (defined as $>3$ databases); however, in $19 \%$ of reviews, authors limited their search to one database. Medline was the most commonly used database (cited by $96 \%$ of reviews). Most (73\%) reviews reported undertaking hand-searching. An extensive search period (defined as $>10$ years) was covered in $64 \%$ of reviews. Language was unrestricted in $29 \%$ of reviews, while the grey literature was reported as searched in just $27 \%$ of reviews. Most $(80 \%)$ reviews presented key words used in the search or the actual search terms for at least one database. While a large majority ( $90 \%$ ) of reviews cited ex-ante study inclusion or exclusion criteria, less than half (48\%) fully described the screening, text review, and selection processes (though an additional $19 \%$ provided partial description of at least one element of these processes). All relevant study designs were deemed to be included in $70 \%$ of reviews. Efforts to avoid overlapping populations were described in $42 \%$ of reviews. A small minority of reviews excluded studies due to low power (14\%) or poor methods (18\%). A PRISMA-type study selection flow diagram was provided in $38 \%$ of studies.
Study selection and/or data extraction was reported as being done by two or more independent reviewers in over half ( $54 \%$ ) of reviews; in $42 \%$ of SRMAs, no statement was made regarding the number of reviewers. All 48 reviews provided effect point estimates along with measures of variance extracted from underlying studies. In nearly all (92\%) reviews, data on major underlying study covariates were also extracted. In a majority $(71 \%)$ of reviews, extracted point estimates were adjusted for commonly reported covariates; however, these often varied across studies. Calculations performed by review authors to derive comparable effect estimates were described in $65 \%$ of reviews. Information on health outcome ascertainment and exposure measurement was extracted by most (79\%) reviews. Authors of underlying studies were contacted for additional information in the case of $27 \%$ of reviews, and $13 \%$ of reviews described using purpose-designed data extraction forms.

Methods The large majority of reviews (94\%) reported using a statistical test of heterogeneity, most commonly the $I^{2}$
Fig. 2 Systematic review of environmental health (EH) epidemiology systematic review and meta-analyses (SRMA): EH SRMAs by publication year and chemical category

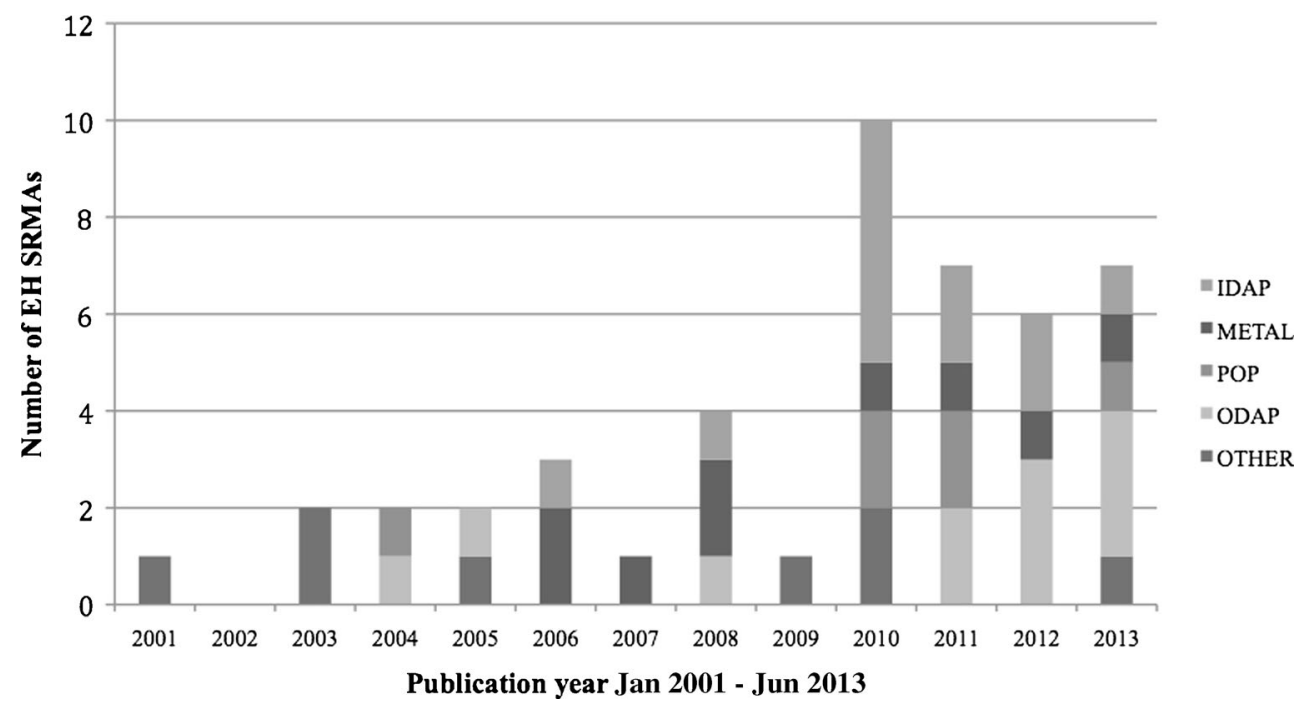


statistic. Moderate to substantial heterogeneity (e.g., $I^{2}>50 \%$ ) was found in more than half of SRMAs. Most reviews employed a random-effect model in the presence of observed heterogeneity. Sources of heterogeneity were explored through stratification and/or meta-regression in $71 \%$ of reviews. We assessed health outcomes of underlying studies to be the same or substantially similar (e.g., same ICD code or same definition and ascertainment process) in $83 \%$ of reviews and similar enough to be reasonably compared (e.g., similar definition and ascertainment process) in an additional $13 \%$. Outcome ascertainment relied predominantly on self-report in nearly one third of reviews.

We assessed exposure metrics to be the same or substantially similar (e.g., the same chemical and measurement technique, with similar exposure conditions) in $31 \%$ of studies and similar enough to be reasonably compared (the same or similar chemical, similar measurement technique, and similar exposure conditions) in an additional $65 \%$ of reviews. In the case of $40 \%$ of reviews, efforts had been made to convert nonsimilar to similar exposure metrics based on clearly stated assumptions. In addition, based on Blair et al. (1995) our checklist identified four desirable exposure metric characteristics, i.e., that measures preferably be (i) direct (rather than proxy); (ii) individual; (iii) quantitative; and (iv) not based on self-report. Our assessment of exposure measurement characteristics reported in SRMAs was based on the preponderance of evidence (i.e., greater than half of underlying studies meeting the criteria). Results suggest that exposure measures were primarily direct in $44 \%$ of the 48 SRMAs, individual in $52 \%$, quantitative in $58 \%$, and avoided self-report in $44 \%$. Exposure heterogeneity was raised as a risk-of-bias concern in the Discussion sections of $77 \%$ of reviews.

A formal quality and/or risk-of-bias evaluation was reported to have been undertaken in $38 \%$ of reviews, and the results of such evaluations were reported in $27 \%$ of reviews. One quarter of SRMAs reported using a published guideline or checklist (or an author-modified version) for this purpose. Quality scores were used in $21 \%$ of reviews. Publication bias was evaluated in $71 \%$ of reviews, most commonly (over $40 \%$ of reviews) using a funnel chart. Some evidence of publication bias was found in $31 \%$ of reviews.

Results, Discussion, and Conclusions Nearly all reviews presented characteristics of underlying studies in summary tables (96\%), and most illustrated pooled effects using forest plots $(85 \%)$. Uncertainty estimates were provided along with summary effect measures in all 48 reviews. Most SRMAs either examined one study design only (or predominantly) or stratified and reported results by study design category. Efforts of underlying studies to examine confounding, case selection, and bias were evaluated and discussed in $58 \%$ of reviews and partially discussed in an additional $21 \%$ of reviews. Thirty- five percent of reviews explored reasons for observed negative or no-effect findings in underlying studies.

Limitations were substantially discussed in $77 \%$ of reviews. Sources of bias and error were raised in this context in $65 \%$ of reviews, while exposure heterogeneity was raised as a limitation in $77 \%$ of reviews. Future EH research recommendations were made in $96 \%$ of reviews. On the other hand, generalizability was discussed in just $33 \%$ of reviews, while EH practice, policy, and improved study-reporting recommendations were made in only 21,25 , and $19 \%$ of reviews.

\section{Discussion}

In this systematic review of EH SRMAs published through mid-2013 comparing methods employed to available guidelines, we found 48 studies using both SR and MA techniques examining associations of low-dose environmental chemicals and adverse health outcomes in general populations. The majority of these reviews were published since 2009, indicating rapid recent uptake of SRMA methods in EH. Nearly half of reviews examined associations between air pollution (indoor and outdoor) exposures and respiratory or CVD outcomes. Over one quarter evaluated associations between various chemical exposures and cancer outcomes. In contrast, we found few SRMAs for many metals (except for arsenic and lead) and POPs, and none for chemicals of more recent interest such as endocrine disruptors. Neurological, immune, and endocrine-related outcomes and upstream effects were also infrequently addressed. Many SRMAs noted that underlying studies were largely from high-income countries (although several covered China only and studies of indoor air pollution were often in developing countries). These findings are broadly consistent with a recent WHO review aimed at estimating the disease burden from chemicals, which found that the largest documentable burden was due to indoor solid fuel smoke and outdoor air pollution (along with secondhand tobacco smoke, not included in our review), but concluded that many chemicals with known health effects could not be included due to lack of meta-analyses providing pooled estimates [2]. High-quality epidemiologic studies and meta-analyses on those missing chemicals are needed in order to more accurately estimate the burden of disease related to environmental exposures.

Reported pooled results were mainly positive and statistically significant. Although some observed associations were not large in magnitude, in many cases strong consistency was noted across numerous studies and dose-response relationships were observed, increasing confidence in the results. Moreover, the risks often involved very large potentially exposed populations: Solid fuels are estimated to be used by half the world's population; outdoor air pollution affects hundreds 
of millions of urban dwellers globally, and disinfection byproducts and arsenic in water supplies affect millions.

The techniques of SRMA can be useful in synthesizing scientific information provided study goals are well-defined and the methods used are sound [13]. In this review comparing actual use of SRMA methods in EH with available guidelines, we found both strengths and weaknesses. Among strengths, we found a high degree of concordance of SRMAs with many elements of general published guidance such as defining ex-ante selection criteria, reporting health outcome ascertainment data and combining health outcomes that were largely comparable, testing for heterogeneity among studies using a statistical test, appropriately combining using a random-effect model in the presence of heterogeneity, and providing forest plots illustrating pooled effects. Most reviews pursued to some extent unbundling of heterogeneity sources across studies, either through stratification or meta-regression, although in many cases, this was limited. Most discussed some limitations, including causes of heterogeneity, error and bias, and most evaluated publication bias. Nearly all provided recommendations for future research.

However, we also identified reporting and methodological shortcomings in the EH SRMA literature reviewed that we divide into two broad categories: (i) weaknesses with respect to general SRMA reporting guidelines, including up-front problem formulation and implications for generalizability and policy; and in search, selection and extraction methods; and (ii) weaknesses in methods reflecting specificities of $\mathrm{EH}$, including exposure measurement, and evaluation of other quality and risk-of-bias parameters, particularly confounders.

\section{General SRMA Guidelines}

Goal Statement and Implications One of the principle requirements of an SRMA is identification of a well-defined research question, while among key SRMA features are their ability to establish whether findings are consistent and generalizable across populations, and to provide an evidence base for policy development. Some SRMA did provide welldefined goal statements and discuss generalizability, including subsequent relevance for policy and practice. For example, in the context of solid fuel IAP, one review identified at the outset childhood respiratory infection prevention strategies and concluded that, despite the review's finding of observed heterogeneity in IAP exposure and study designs, consistent mounting evidence warranted their enhanced application [19]. However, we found that the large majority of SRMAs did not do so. Strong, clear goal statements were present in just over half of reviews. Generalizability of findings to other populations, and implications for policy, were discussed in a small minority of reviews. EH SRMAs provide an opportunity for enhancing policy and practice relevance of science findings at a time when the field of EH risk assessment is shifting toward greater attention to up-front problem formulation and its integration with policy- and decision-making $[15 \bullet, 67]$. This opportunity is currently under-utilized.

Search, Selection, and Extraction While searches were generally well reported, their scope was often limited to one or two databases, language was often restricted, and time horizons were in some cases short. Unpublished materials were sought in less than one third of reviews, while research suggests that the grey literature is important in identifying studies [68]. This suggests that some EH SRMAs may be biased toward recent English language studies indexed in Medline. This is also reflected in the finding that approximately half of the reviews that examined publication bias found some evidence of it. Increased use of databases available internationally and eliminating language restrictions (particularly with the goal of identifying studies in low- and middle-income countries) are recommended. In addition, while peer review helps ensure high-quality SRMAs, examination of the grey literature can help identify relevant peer-reviewed papers. Less than half of reviews described procedures to avoid overlapping populations or provided a PRISMA-recommended flow diagram, suggesting some lack of transparency and replicability in study selection. Journal space limitations are likely partly responsible, as such features may have been undertaken and not reported. A solution employed by several SMRAs [34, 36] is providing standard SRMA elements as supplemental material. Nearly half of reviews did not report the recommended two or more independent reviewers while use of piloted extraction forms was reported by a minority of studies, suggesting potential for risk of error in data extraction. This also raises the issue of SRMA resource intensity. Alternatives such as an error-check procedure [20] or a second independent reviewer extracting a random sample of studies [69] may be options to explore. While their validity has yet to be determined, such approaches may help address the trade-off between limited resources and risk of error.

\section{SRMA Guidelines Specific to EH}

Exposure Measurement Exposure characterization was reported to be a key source of between-study heterogeneity in the majority of SRMAs. Although nearly all reviews combined underlying studies whose exposure metrics were largely similar, these metrics were deemed not fully comparable in nearly two thirds of reviews. Exposure metrics commonly used indirect proxies and relied on non-individual measures and self-report, with OAP and metal exposure characterizations more broadly consistent with recommendations than the other three chemical categories. Only a few reviews discussed the likely direction of potential exposure-related error or bias; such insights are useful additions to SRMAs. Some studies not included in this review have concluded that exposure 
heterogeneity was sufficient to make combining unadvisable, e.g., in the case of risk assessment for PCBs [70]. The lack of updated, widely endorsed EH-specific guidelines for SRMA may be one factor in the observed inconsistency in exposure metrics; a well-disseminated EH SRMA consensus reporting statement with clear principles regarding exposure measurement would be an important contribution. These findings also highlight the potential role for SRMAs in recommending standard practices for exposure measurement protocols in underlying studies. While several SRMAs provided such recommendations [27, 47], this opportunity is under-utilized.

Other Sources of Bias While most reviews extracted data on the covariates adjusted for in underlying studies and reported adjusted rather than crude effect estimates, nearly one quarter did not clearly identify potential confounders, and in most reviews underlying studies adjusted for sets of confounders that differed (e.g., key confounders such as smoking status were often missing). More broadly, quality (standards and reporting) and/or risk-of-bias (internal validity based on study design considerations) evaluations were undertaken in less than $40 \%$ of reviews (though the percentage increased over the time-period examined). Many additional SRMAs examined specific quality- and bias-related issues in their Discussion sections; however, without the structure of a formalized quality evaluation, the analysis may be less transparent, systematic, and thorough. Quality scores, discouraged in guidelines for their potential to add subjective bias, were used in half of quality evaluations undertaken, and $11 \%$ percent of SRMAs reported performing quality assessments but did not report their findings. Journal space constraints may have contributed to the latter outcome, and use of supplemental information files may be a partial solution. As in the case of exposure measurement, clear EH-specific standards for reporting quality and risk-ofbias evaluations would be a substantial contribution.

This is the first study to our knowledge to systematically review the EH SRMA literature comparing methods used in practice with guidance documents. A similar review of SRMAs in the occupational health $(\mathrm{OH})$ field identified 60 OH SRMAs examining mainly cancer outcomes and found limitations and inconsistencies in exposure characterization and inadequate and unclear adjustment for confounders [71], findings generally consistent with ours. Unlike our study, that review found many OH SRMAs used fixed effects models even in the presence of substantial statistically confirmed heterogeneity. A review of SRMA in psychiatric epidemiology found substantial heterogeneity among studies and noted particularly wide variety and poor comparability of outcome measurement instruments [72], while a recent review of riskof-bias assessments in epidemiological studies found that assessment conclusions were often poorly integrated into study findings [73], both broadly consistent with our results.

Our meta-review is subject to certain limitations. Our search may not have identified all published EH SRMAs during the search period; however, it was designed to cast a wide net and incorporated several strategies to maximize location of all relevant studies. Our quality review checklist was derived from guidelines available during the period of our search. In order to derive a checklist of reasonable length, we left out elements that could have been included; however, we believe that a slightly different list of items is likely to have resulted in similar overall findings. Because the SRMAs in our study covered a wide range of chemical exposures and health outcomes, our findings are at a high level of aggregation; focus on more specific exposure-outcome associations would allow for more in-depth evaluation. One such review of methods and reporting in 16 SRMAs of $\mathrm{EH}$ and $\mathrm{OH}$ exposures and pregnancy outcomes identified shortcomings primarily related to exposure misclassification and confounding [74], aligning broadly with our findings.

In addition, our study was limited to epidemiological studies in humans. In EH, many decisions are driven by animalbased and cellular-based toxicology experiments, usually in contexts where human studies are limited and/or difficult to carry out. While a few SRMAs reviewed both the toxicology and epidemiology literature [27], the vast majority did not. It was beyond the scope of this review to examine the toxicological literature; however, one such review found that while use of SR methods was adequate, application of MA methods was weak [75]; an updated animal review would be useful in the future, making use of the reporting tools and risk-of-bias criteria currently under development [76].

Our review is broadly consistent with recent efforts at refinement and development of EH SRMA tools by NIEHS/ NTP [14•], EPA/IRIS [15•], and the Navigation Guide [17•]. Among the aims of these newer approaches is to go a step beyond evaluating reporting quality to designating a confidence level for EH evidence, based on the strength of inherent underlying study design, with adjustments made for factors that reduce confidence (such as presence of bias in exposure measurement and potential unadjusted confounding) and factors that enhance confidence (such as existence of doseresponse relationships, consistency in observed effects, and large magnitudes of effect) [14•]. Our study findings support these efforts by demonstrating gaps and shortcomings in exposure and risk-of-bias assessment in the published literature and by suggesting the contribution to improvements in these areas that could be made by tailored EH SRMA guidelines. It was not the goal of our study to conclude with strength-ofevidence findings for published EH SRMAs; however, use of the emerging tools for this purpose could add to the literature. 


\section{Conclusion}

In this meta-review of published EH SRMAs, we found methods and reporting largely conform to available general guidelines. However, there were important exceptions (including in problem formulation, policy implications, and search, selection, and extraction); moreover, we found that SRMAs reflected lower awareness of and poorer conformity with available guidelines applicable to EH studies (particularly exposure measurement and more general risk-of-bias assessment). Many journals have incorporated PRISMA and MOOSE statements into their author guidelines. As a continuation of refinements to SRMA methods for observational studies, we recommend development of a consensus statement of definitive guidelines for EH SRMAs that is published, welldisseminated, and adopted by journals as guidance to study authors and peer reviewers.

Even with the design, methods, and reporting weaknesses observed, the EH SRMAs examined in this review point to broad trends in the EH evidence base, including policy priorities involving large populations (e.g., risk of lung cancer, COPD, and child respiratory infection associated with indoor solid fuel smoke exposure; CVD risks associated with outdoor $\mathrm{PM}_{2.5}$ exposure and arsenic); areas of apparent lack of significant associations (e.g., DDE and breast cancer; disinfection by-products, and some birth outcomes); and gaps in the evidence base for certain chemicals and health outcomes (e.g., endocrine disruptors, some metals, and POPs) and populations (e.g., low- and middle-income countries for exposures different from indoor air pollution). We recommend a regularly updated meta-review of the EH epidemiological SRMA literature with the goal of identifying trends in evidence (including strength-of-evidence assessments applying the emerging tools), identifying gaps and research priorities, and stocktaking of methods and reporting quality.

Acknowledgments The authors thank the Risk Sciences and Public Policy Institute of the Johns Hopkins Bloomberg School of Public Health for supporting this project and Dr. Ramya Chari for her contribution to conceptualizing the work.

\section{Compliance with Ethics Guidelines}

Conflict of Interest Mary C. Sheehan and Juleen Lam declare that they have no conflict of interest.

Human and Animal Rights and Informed Consent This article does not contain any studies with human or animal subjects performed by any of the authors.

Open Access This article is distributed under the terms of the Creative Commons Attribution 4.0 International License (http:// creativecommons.org/licenses/by/4.0/), which permits unrestricted use, distribution, and reproduction in any medium, provided you give appropriate credit to the original author(s) and the source, provide a link to the Creative Commons license, and indicate if changes were made.

\section{References}

Papers of particular interest, published recently, have been highlighted as:

- Of importance

1. World Health Organization (WHO). Preventing disease through healthy environments. Towards an estimate of the environmental burden of disease. 2006 Available: http://www.who.int/ quantifying_ehimpacts/publications/preventingdisease.pdf [accessed 25 March 2015]

2. Pruss-Ustun A, Vickers C, Haefliger P, Bertollini R. Knowns and unknowns on burden of disease to chemicals: a systematic review. En Health. 2011;10:9. doi:10.1186/1476-069X-10-9.

3. Bellinger D. A strategy for comparing the contributions of environmental chemicals and other risk factors to children's neurodevelopment. Environ Health Perspect. 2012;120(4):501-7.

4. WHO. Health and environment linkages initiative (HELI). World Health Organization/United Nations Development Program. 2014 Available: http://www.who.int/heli/en/ [accessed 17 April 2015]

5. Simera I, Moher D, Hoey J, Schultz KF, Altman DG. A catalogue of reporting guidelines for health research. Eur J Clin Investig. 2010;40:35-52.

6. Woodruff TJ, Sutton P. The navigation guide systematic review methodology: a rigorous and transparent method for translating environmental health science into better health outcomes. Environ Health Perspect. 2011;122(10):1007-14.

7. Moher D, Liberati A, Tetzlaff J, Altman D, Group PRISMA. Preferred reporting items for systematic reviews and meta-analyses: the PRISMA statement. PLoS Med. 2009;6(7), e100097. doi:10. 1371/journal.pmed.1000097.

8. Dickersin K. Health care policy: to reform US health care, start with systematic review. Science. 2010;329(5991):516-7.

9. Jones DR. Meta-analysis of observational studies: a review. J R Soc Med. 1992;85(3):165-8.

10. Thompson SG, Higgins JP. How should meta-regression analyses be undertaken and interpreted? Stat Med. 2002;21:1559-73. doi:10. 1002/sim.1187.

11. Sanderson S, Tatt ID, Higgins JPT. Tools for assessing quality and susceptibility to bias in observational studies in epidemiology: a systematic review and annotated bibliography. Int J Epidemiol. 2007;36(3):666-76.

12. Stroup DF, Berlin JA, Morton SC, Olkin I, Williamson GD, Rennie $\mathrm{D}$, et al. Meta-analysis of observational epidemiology: a proposal for reporting. JAMA. 2000;283(15):2008-12.

13. Moher D, Shekelle PG, Stewart LA. 2012. Establishing a new journal for systematic review products. Syst Review 1(1) doi: 10.1186/ 2046-4053-1-1

14. Rooney AA, Boyles AL, Wolfe MS, Buchier JR, Thayer KA. Systematic review and evidence integration for literature-based environmental health science assessments. Environ Health Perspect. 2014;122(7):711-8. Presents a seven-step framework for systematic review and evidence integration for reaching hazard identification conclusions in NTP/OHAT assessments, including rating the body of evidence (published after the period of review for the present study). 
15. Council NR. Review of the EPA's integrated risk information system (IRIS) process. Washington: National Academies Press; 2014. Presents findings of the NRC Committee to Review the IRIS process, concludes that systematic review methods would substantially improve the IRIS process, and uses SR methods to evaluate the EPA's ongoing changes to IRIS based on recommendations made in the context of the 2011 NRC Formaldehyde Assessment.

16. Lam J, Koustas E, Sutton P, Johnson PI, Atchley DS, Sen S, et al. The navigation guide: evidence-based medicine meets environmental health: integration of animal and human evidence for PFOA effect on fetal growth. Environ Health Perspect. 2014;122(10): 1040-51.

17. Woodruff TJ, Sutton P. The navigation guide systematic review methodology: a rigorous and transparent method for translating environmental health science into better health outcomes. Environ Health Perspect. 2014;122(10):1007-14. Outlines the Navigation Guide, a proof-of-concept for new EH SRMA methods aimed at reducing bias and enhancing transparency in use of these tools, and developing a strength-of-evidence evaluation protocol based on the Grading of Recommendations, Assessment, Development and Evaluations (GRADE). (Published after the period of review for the present study.).

18. Blair A, Burg J, Foran J, Gibb H, Greenland S, Morris R, et al. Guidelines for the application of meta-analysis in environmental epidemiology. Reg Tox Pharm. 1995;22:189-97.

19. Dherani M, Pope D, Mascarenhas M, Smith KR, Weber M, Bruce N. Indoor air pollution from unprocessed solid fuel use and pneumonia risk in children under five years: a systematic review and meta-analysis. Bull World Health Org. 2008;86(5):390-8.

20. Hosgood HD, Wei H, Sapkota A, Choudhury I, Bruce N, Smith KR, et al. Household coal use and lung cancer: systematic review and meta-analysis of case-control studies, with an emphasis on geographic variation. Int J Epidemiol. 2011;40:719-28.

21. Hu G, Zhou Y, Tian J, Yao W, Li J, Li B, et al. Risk of COPD from exposure to biomass smoke: a meta-analysis. Chest. 2010;138(1): 20-31.

22. Kurmi OP, Semple S, Simkhada P, Smith WCS, Ayres J. COPD and chronic bronchitis risk of indoor air pollution from solid fuel: a systematic review and meta-analysis. Thorax. 2010;65:221-8.

23. Kurmi OP, Arya PH, Lam K-BH, Sorahan T, Ayres JG. Lung cancer risk and solid fuel smoke exposure: a systematic review and metaanalysis. Eur Respir J. 2012;40:1228-37.

24. Misra P, Srivastava R, Krishnan A, Sreenivaas V, Pandav CS. Indoor air pollution-related acute lower respiratory infections and low birthweight: a systematic review. J Trop Pediatrics. 2012;58(6): 457-66.

25. Po JYT, FitzGerald JM, Carlsten C. Respiratory disease associated with solid biomass fuel exposure in rural women and children: a systematic review and meta-analysis. Thorax. 2011;66:232-9.

26. Pope DP, Mishra V, Thompson L, Siddiqui AR, Rehfuess EA, Weber M. Risk of low birth weight and stillbirth associated with indoor air pollution from solid fuel use in developing countries. Epidemiol Rev. 2010;32:70-81.

27. Sumpter C, Chandramohan D. Systematic review and metaanalysis of the associations between indoor air pollution and tuberculosis. Trop Med Int Health. 2013;18(1):101-8.

28. Zhao Y, Wang S, Aunan K, Seip HM, Hao J. Air pollution and lung cancer risks in China: a meta-analysis. Sci Tot Environ. 2006;366: 500-13.

29. McGwin G, Lienert J, Kenndy JI. Formaldehyde exposure and asthma in children: a systematic review. Environ Health Perspect. 2010;118:313-7.

30. Chen H, Goldberg MS, Villeneuve PJ. A systematic review of the relation between long-term exposure to ambient air pollution and chronic diseases. Rev Environ Health. 2008;23(4):243-98.
31. Hoek G, Krishnan RM, Beelen R, Peters A, Ostro B, Brunekreef B, et al. Long term air pollution exposure and cardio-respiratory mortality: a review. Environ Health. 2013;12:43. doi:10.1186/1476069X-12-43.

32. Ito K, de Leon SF, Lippmann M. Associations between ozone and daily mortality: analysis and meta-analysis. Epidemiol. 2005;16(4): 446-57.

33. Li X-Y, Yu X-B, Liang W-W, Yu N, Wang L, Ye X-J, et al. Metaanalysis of association between particulate matter and stroke attack. CNS Neurosci Ther. 2012;18:501-8.

34. Mustafic H, Jabre P, Caussin C, Murad MH, Escolano S, Tafflet M, et al. Main air pollutants and myocardial infarction: a systematic review and meta-analysis. JAMA. 2012;307(7):713-21.

35. Pieters N, Plusquin M, Cox B, Kicinski M, Vangronsveld J, Nawrot TS. An epidemiological appraisal of the association between heart rate variability and particular air pollution: a meta-analysis. Heart. 2012;98:1127-35.

36. Shah ASV, Langrish JP, Nair H, McAllister DA, Hunter AL, Donaldson K, et al. Global association of air pollution and heart failure: a systematic review and meta-analysis. Lancet. 2013;382(9897):1039-48.

37. Shang Y, Sun Z, Cao J, Wang X, Zhong L, Bi X, et al. Systematic review of Chinese studies of short-term exposure to air pollution and daily mortality. Environ Int. 2013;54:100-11.

38. Steib DM, Chen L, Eshoul M, Judek S. Ambient air pollution, birth weight and pre-term birth: a systematic review and meta-analysis. Environ Res. 2012;117:100-11.

39. Vrijheid M, Martinez D, Manzanares S, Dadvand P, Schembari A, Rankin J, et al. Ambient air pollution and risk of congenital anomalies: a systematic review and meta-analysis. Environ Health Perspect. 2011;119:598-606.

40. Ward DJ, Ayres JG. Particulate air pollution and panel studies in children: a systematic review. Occup Environ Med. 2004;61, e13. doi:10.1136/oem.2003.007088.

41. Weinmayr G, Romeo E, de Sario M, Weiland SK, Forastiere F. Short term effects of $\mathrm{PM}_{10}$ and $\mathrm{NO}_{2}$ on respiratory health among children with asthma or asthma-like symptoms: a systematic review and meta-analysis. 2010

42. Abhyankar LN, Jones MR, Guallar E, Navas-Acien A. Arsenic exposure and hypertension: a systematic review. Environ Health Perspect. 2011;120(4):494-500.

43. Aminzadeh KK, Etminan M. Dental amalgam and multiple sclerosis: a systematic review and meta-analysis. J Public Health Dent. 2007;67(1):64-6.

44. Gallagher CM, Meliker JR. Blood and urine cadmium, blood pressure, and hypertension: a systematic review and meta-analysis. Environ Health Perspect. 2010;118:1676-84.

45. Chu H-A, Crawford-Brown DJ. Inorganic arsenic in drinking water and bladder cancer: a meta-analysis for dose-response assessment. Int J Environ Res Public Health. 2006;3(4):316-22.

46. Mink PJ, Alexander DD, Barraj LM, Kelsh MA, Tsuji JS. Lowlevel arsenic exposure in drinking water and bladder cancer: review and meta-analysis. Reg Toxicol Pharmacol. 2008;52:299-310.

47. Moon K, Guallar E, Navas-Acien A. Arsenic exposure and cardiovascular disease: an updated systematic review. Curr Atheroscler Rep. 2012;14:542-55.

48. Navas-Acien A, Silbergeld EK, Streeter RA, Clark JM, Burke TA, Guallar E. Arsenic exposure and type 2 diabetes: a systematic review of the experimental and epidemiological evidence. Environ Health Perspect. 2006;114:641-8.

49. Navas-Acien A, Schwartz BS, Rothenberg SJ, Hu H, Silbergeld EK, Guallar E. Bone lead levels and blood pressure endpoints: a meta-analysis. Epidemiol. 2008;19:496-504.

50. Pocock S, Smith M, Baghurst P. Environmental lead and children's intelligence: a systematic review of the epidemiological evidence. BMJ. 1994;309:1189-97. 
51. Rodriguez-Barranco M, Lascana M, Aguilar-Garduno C, Alguacil $\mathrm{J}$, Gil F, Gonzalez-Alzaga B, et al. Association of arsenic, cadmium and manganese exposure with neurodevelopment and behavioural disorders in children: a systematic review and meta-analysis. Sci Tot Environ. 2013;454-455:562-77.

52. Allen MT, Levy LS. Parkinson's disease and pesticide exposure: a new assessment. Crit Rev Toxicol. 2013;46(6):515-34.

53. Bailey HD, Armstrong BK, de Klerk NH, Fritschi N, Attia J, Scott RJ, et al. Exposure to professional pest control treatments and the risk of childhood acute lymphoblastic leukemia. Int J Cancer. 2011;129:1678-168.

54. Lopez-Cervantes M, Torres-Sanchez L, Tobias A, Lopez-Carillo L. Dichlorodiphenyldichloroethane burden and breast cancer risk. Environ Health Perspect. 2004;112:207-14.

55. Priyadarshi A, Khuder SA, Shaub EA, Priyadarshi SS. Environmental risk factors and Parkinson's disease: a metaanalysis. Environ Res Sect A. 2001;86:122-7.

56. Turner MC, Wigle DT, Krewski D. Residential pesticides and childhood leukemia: a systematic review and meta-analysis. Environ Health Perspect. 2010;118:33-41.

57. Van Maele-Fabry G, Lantin A-C, Hoet P, Lison D. Residential exposure to pesticides and childhood leukaemia: a systematic review and meta-analysis. Environ Int. 2011;37:280-91.

58. Wu H, Bertrand K, Choi AL, Hu FB, Laden F, Grandjean P, et al. Persistent organic pollutants and type 2 diabetes: a prospective analysis in the nurses' health study and meta-analysis. Environ Health Perspect. 2013;121:153-61.

59. Choi AL, Sun G, Zhang Y, Grandjean P. Developmental fluoride neurotoxicity: a systematic review and meta-analysis. Environ Health Perspect. 2013;120:1362-8.

60. Grellier J, Bennett J, Patelarou E, Smith RB, Toledano MB, Rushton L, et al. Exposure to disinfection by-products, fetal growth and prematurity: a systematic review and meta-analysis. Epidemiol. 2010;21(3):300-13.

61. Hwang B-F, Jaakkola JJ. Water chlorination and birth defects: a systematic review and meta-analysis. Arch Environ Health. 2003;58(2):83-91.

62. Morris RD, Audet A-M, Angelillo IF, Chalmers TC, Mosteller F. Chlorination, chlorination by-products and cancer: a meta-analysis. Am J Public Health. 1992;82(7):955-63.

63. Nieuwenhuijsen MJ, Martinez D, Grellier J, Bennett J, Best N, Iszatt $\mathrm{N}$, et al. Chlorination disinfection by-products in drinking water and congenital anomalies: review and meta-analysis. Environ Health Perspect. 2009;117:1486-93.

64. Rahman MB, Driscoll T, Cowie C, Armstrong BK. Disinfection byproducts in drinking water and colorectal cancer: a meta-analysis. Int J Epidemiol. 2010;39:733-45.
65. Takkouche B, Etiminan M, Montes-Martinez A. Personal use of hair dyes and risk of cancer: a meta-analysis. JAMA. 2005;293(20):2516-25.

66. Villanueva CM, Fernandez F, Malats N, Grimalt JO, Kogevinas M. Meta-analysis of studies on individual consumption of chlorinated drinking water and bladder cancer. J Epidemiol Community Health. 2003;57:166-73.

67. Council NR. Science and decisions: advancing risk assessment. Washington: National Academies Press; 2009.

68. Woodman JL, Harden A, Thomas J, Brunton J, Kavanaugh J, Stansfield C. Searching for systematic reviews of the effects of social and environmental interventions: a case study of children and obesity. J Med Libr Assoc. 2010;98(2):140-6.

69. Jones-Hughes T, Peters J, Whear R, Cooper C, Evans H, Depledge $\mathrm{M}$, et al. Are interventions to reduce the impact of arsenic contamination in groundwater effective? a systematic review. Environmental Evidence. 2013;2:11. doi:10.1186/2047-2382-2-11.

70. Goodman M, Squibb K, Youngstrom E, Anthony LG, Kenworthy L, Lipkin PH. Using systematic reviews and meta-analyses to support regulatory decision making for neurotoxicants: lessons learned from a case study of PCBs. Environ Health Perspect. 2010;118: 727-34.

71. McElvenny DM, Armstrong BG, Jarup L, Higgins JPT. Metaanalysis in occupational epidemiology: a review of practice. Occup Med. 2004;54:336-44.

72. Brugha TS, Matthews R, Morgan Z, Hill T, Alonso J, Jones DR. Methodology and reporting of systematic reviews and metaanalyses of observational studies in psychiatric epidemiology: a systematic review. Br J Psychiatry. 2012;200(6):44653 .

73. Katikireddi SV, Egan M, Petticrew M. How do systematic reviews incorporate risk of bias assessments into the synthesis of evidence? A methodological study. J Epidemiol Community Health. 2015;69(2):189-95.

74. Nieuwenhuijsen M, Dadvand P, Grellier J, Martinez D, Vrijheid M. Environmental risk factors of pregnancy outcomes: a summary of recent meta-analyses of epidemiological studies. Environ Health. 2013;12:6. doi:10.1186/1476-069X-12-6.

75. Peters JL, Sutton AJ, Jones DR, Rushton L, Abrams KR. A systematic review of systematic reviews and meta-analyses of animal experiments with guidelines for reporting. J Environ Sci Health. 2006;41(7):1245-58.

76. Krauth D, Woodruff $\mathrm{T}$, Bero L. Instruments for assessing risk of bias and other methodological criteria of published animal studies: a systematic review. Environ Health Perspect. 2013;121(9):985-92. 